\title{
Lithium Amide-Assisted Asymmetric Mannich-Type Reactions of Menthyl Acetate with PMP-Aldimines
}

\author{
Seiji Hata, Mayu Iguchi, Tetsuo Iwasawa, Ken-ichi Yamada, and Kiyoshi Tomioka*
}

Graduate School of Pharmaceutical Sciences, Kyoto University

Yoshida, Sakyo-ku, Kyoto 606-8501, Japan

E-mail: tomioka@pharm.kyoto-u.ac.jp

Extracts were dried over sodium sulfate unless otherwise noted. Purification was carried out by silica gel column chromatography unless otherwise noted. All melting points are uncorrected. IR spectra were expressed in $\mathrm{cm}^{-1} \cdot{ }^{1} \mathrm{H}$ and ${ }^{13} \mathrm{C}$ NMR spectra were taken in $\mathrm{CDCl}_{3}$. Chemical shift values are expressed in ppm relative to internal tetramethylsilane. Abbreviations are as follows: $s$, singlet; d, doublet; t, triplet; q, quartet; m, multiplet; br, broad.

\section{Acetic acid L-menthyl ester (1):}<smiles>CC(=O)OC1CCCCC1C(C)C</smiles>

to a solution of L-menthol $(25.0 \mathrm{~g}, 0.16 \mathrm{~mol})$ and DMAP $(1.9 \mathrm{~g}, 0.016 \mathrm{mmol})$ in pyridine $(19 \mathrm{~mL})$ was added acetic anhydride $(32.6 \mathrm{~g}, 0.32 \mathrm{~mol})$ dropwise over $3 \mathrm{~min}$. After being stirred at room temperature for $1 \mathrm{~h}$, the reaction mixture was quenched with ice water $(80 \mathrm{~mL})$ and extracted with $\mathrm{Et}_{2} \mathrm{O}(200 \mathrm{~mL}, 100 \mathrm{~mL}$ x 2). The combined organic layers were washed with $0.5 \mathrm{M}$ $\mathrm{HCl}(100 \mathrm{~mL} \times 3)$, water $(80 \mathrm{~mL})$, sat. $\mathrm{NaHCO}_{3}(80 \mathrm{~mL})$, and brine $(80 \mathrm{~mL})$, and then dried. Concentration gave a colorless oil (33.2 g). Purification by fractional distillation (bp $119-120{ }^{\circ} \mathrm{C} / 26$ $\mathrm{mmHg})$ gave $1(23.3 \mathrm{~g}, 74 \%)$ as a colorless oil of $[\alpha]^{20}{ }_{\mathrm{D}}-82.2\left(c\right.$ 8.10, $\left.\mathrm{C}_{6} \mathrm{H}_{6}\right) .{ }^{1} \mathrm{H}$ NMR: $0.76(3 \mathrm{H}, \mathrm{d}$, $J=7.0 \mathrm{~Hz}), 0.88(1 \mathrm{H}, \mathrm{m}), 0.89(3 \mathrm{H}, \mathrm{d}, J=3.4 \mathrm{~Hz}), 0.91(3 \mathrm{H}, \mathrm{d}, J=2.8 \mathrm{~Hz}), 0.96(1 \mathrm{H}, \mathrm{m}), 1.06(1 \mathrm{H}$, m), $1.34(1 \mathrm{H}, \mathrm{m}), 1.48(1 \mathrm{H}, \mathrm{m}), 1.67(2 \mathrm{H}, \mathrm{m}), 1.87(1 \mathrm{H}, \mathrm{m}), 1.99(1 \mathrm{H}, \mathrm{m}), 2.05(3 \mathrm{H}, \mathrm{s}, \mathrm{Me}), 4.67$ (1H, ddd, $J=11,11,4.3 \mathrm{~Hz}) .{ }^{13} \mathrm{C}$ NMR: 16.3, 20.7, 21.2, 21.9, 23.4, 26.2, 31.3, 34.2, 40.9, 46.9, 
74.0, 170.6. IR (neat): 1730. CIMS m/z: $198\left(\mathrm{M}^{+}\right)$. Anal. Calcd for $\mathrm{C}_{14} \mathrm{H}_{26} \mathrm{O}_{4}: \mathrm{C} 72.68, \mathrm{H} 11.18$. Found: C 72.52, H 11.44.

Asymmetric Mannich-Type Reactions. (1R,2S,5R)-2-Isopropyl-5-methylcyclohexyl 3-(4-methoxyphenylamino)-3-phenylpropanoate (4a):<smiles>COc1ccc(NC(CC(=O)OC2C[C@H](C)CCC2C(C)C)c2ccccc2)cc1</smiles>

a solution of L-menthyl acetate $(794 \mathrm{mg}, 4.0 \mathrm{mmol})$ in THF $(6 \mathrm{~mL})$ was added dropwise over 5 min to a solution of LDA $(9.0 \mathrm{mmol})$ in THF $(6 \mathrm{~mL})$ at $-78{ }^{\circ} \mathrm{C}$, and the resulting mixture was stirred at $-78{ }^{\circ} \mathrm{C}$ for $0.5 \mathrm{~h}$. A solution of imine $3 \mathbf{a}$ ( $423 \mathrm{mg}, 2.0 \mathrm{mmol}$ ) in THF $(6 \mathrm{~mL})$ was added dropwise over $10 \mathrm{~min}$ to the mixture at $-78{ }^{\circ} \mathrm{C}$, and the mixture was stirred at $-30{ }^{\circ} \mathrm{C}$ for $4 \mathrm{~h}$. The reaction was quenched with sat. $\mathrm{NH}_{4} \mathrm{Cl}(20 \mathrm{~mL})$ at $-78{ }^{\circ} \mathrm{C}$ and extracted with AcOEt $(30 \mathrm{~mL}$ x 3). The combined organic layers were washed with brine $(20 \mathrm{~mL})$, and dried. Concentration gave a pale yellow solid (1.30 g). Chromatography (toluene/AcOEt $=200 / 1)$ gave 4a (622 mg, 76\%, 92\% de) as colorless needles. Recrystallization of the diasrereomeric mixture from $\mathrm{MeOH}$ gave a pure major isomer $(70 \%)$ as colorless needles of mp $149.5-150{ }^{\circ} \mathrm{C}$ and $[\alpha]^{25}-38.4$ (c $\left.0.58, \mathrm{CHCl}_{3}\right) .{ }^{1} \mathrm{H}$ MR: $0.57(3 \mathrm{H}, \mathrm{d}, J=7.0 \mathrm{~Hz}), 0.72(3 \mathrm{H}, \mathrm{d}, J=7.0 \mathrm{~Hz}), 0.80-0.91(5 \mathrm{H}, \mathrm{m})$, 0.95-1.02 (1H, m), 1.28-1.32 (1H, m), 1.41-1.44 (1H, m), 1.60-1.66 (3H, m), 1.87-1.89 (1H, m), $2.72(1 \mathrm{H}, \mathrm{dd}, J=8.2,14.7 \mathrm{~Hz}), 2.78(1 \mathrm{H}, \mathrm{dd}, J=5.8,14.7 \mathrm{~Hz}), 3.69(3 \mathrm{H}, \mathrm{s}), 4.24$ (1H, br), 4.66 $(1 \mathrm{H}, \mathrm{ddd}, J=4.3,10.4,10.4 \mathrm{~Hz}), 4.75(1 \mathrm{H}, \mathrm{dd}, J=5.8,8.2 \mathrm{~Hz}), 6.49(2 \mathrm{H}, \mathrm{d}, J=8.8 \mathrm{~Hz}), 6.69(2 \mathrm{H}$, $\mathrm{d}, J=8.8 \mathrm{~Hz}), 7.23(1 \mathrm{H}, \mathrm{m}), 7.29-7.32(2 \mathrm{H}, \mathrm{m}), 7.36-7.38(2 \mathrm{H}, \mathrm{d}, J=7.7 \mathrm{~Hz}) .{ }^{13} \mathrm{C} \mathrm{NMR}: 15.9$, 20.7, 22.0, 23.2, 25.9, 31.4, 34.2, 40.9, 43.5, 47.0, 55.7, 55.9, 74.6, 114.7, 114.8, 126.3, 127.3, 128,7, 140.9, 142.4, 152.2, 170.7. IR (nujol): 3350, 1710. EIMS $m / z: 409\left(\mathrm{M}^{+}\right), 212$. Anal. Calcd for $\mathrm{C}_{26} \mathrm{H}_{35} \mathrm{NO}_{3}$ : C, 76.25; H, 8.61; N, 3.42. Found: C, 76.33; H, 8.48; N, 3.38.

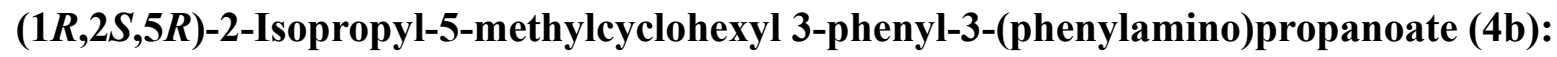

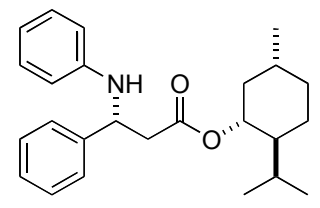

recrystallization of a diastereomeric mixture $(92 \%$ de) from EtOH gave a pure major isomer as colorless needles of $\mathrm{mp} 137-137.5^{\circ} \mathrm{C}$ and $[\alpha]^{25}-41.9\left(c 0.99, \mathrm{CHCl}_{3}\right) .{ }^{1} \mathrm{H}$ NMR: $0.56(3 \mathrm{H}, \mathrm{d}, J=7.0 \mathrm{~Hz}), 0.71(3 \mathrm{H}, \mathrm{d}, J=7.0 \mathrm{~Hz}), 0.83-0.93(5 \mathrm{H}, \mathrm{m}), 0.95-1.05(1 \mathrm{H}, \mathrm{m}), 1.27-1.33$ $(1 \mathrm{H}, \mathrm{m}), 1.40-1.49(1 \mathrm{H}, \mathrm{m}), 1.57-1.68(3 \mathrm{H}, \mathrm{m}), 1.84-1.89(1 \mathrm{H}, \mathrm{m}), 2.75(1 \mathrm{H}, \mathrm{dd}, J=8.3,14.4 \mathrm{~Hz})$, $2.81(1 \mathrm{H}, \mathrm{dd}, J=5.5,14.4 \mathrm{~Hz}), 4.50(1 \mathrm{H}, \mathrm{br}), 4.66(1 \mathrm{H}, \mathrm{ddd}, J=4.6,10.7,10.7 \mathrm{~Hz}), 4.83(1 \mathrm{H}, \mathrm{dd}, J$ $=5.5,8.3 \mathrm{~Hz}), 6.53(2 \mathrm{H}, \mathrm{d}, J=7.9 \mathrm{~Hz}), 6.65(1 \mathrm{H}, \mathrm{t}, J=7.4 \mathrm{~Hz}), 7.09(2 \mathrm{H}, \mathrm{dd}, J=7.4,7.9 \mathrm{~Hz}), 7.23$ $(1 \mathrm{H}, \mathrm{t}, J=7.4 \mathrm{~Hz}), 7.31(2 \mathrm{H}, \mathrm{t}, J=7.4 \mathrm{~Hz}) 7.38(2 \mathrm{H}, \mathrm{d}, J=7.4 \mathrm{~Hz}) .{ }^{13} \mathrm{C}$ NMR: 15.8, 20.6, 21.9, 23.1, 25.8, 31.3, 34.1, 40.8, 43.5, 46.9, 55.0, 74.6, 113.4, 117.6, 126.3, 127.4, 128.7, 129.1, 142.2, 146.7, 170.7. IR (neat): 3391, 1713. EIMS $m / z: 379,303,182$. Anal. Calcd for $\mathrm{C}_{25} \mathrm{H}_{33} \mathrm{NO}_{2}$ : C, 
79.11; H, 8.76; N, 3.69. Found: C, 79.00; H, 8.74; N, 3.66.

\section{(1R,2S,5R)-2-Isopropyl-5-methylcyclohexyl 3-phenyl-3-[4-(trifluoromethyl)- phenylamino]propanoate (4c):}

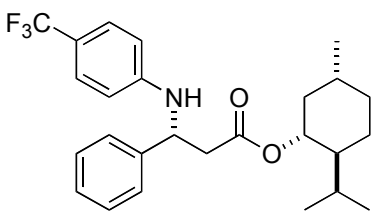

a diastereomeric mixture $\left(60 \%\right.$ de) as colorless needles of mp $142-153{ }^{\circ} \mathrm{C}$ and $[\alpha]^{25}{ }_{\mathrm{D}}-37.9\left(c\right.$ 1.02, $\left.\mathrm{CHCl}_{3}\right) .{ }^{1} \mathrm{H}$ NMR: $0.56(2.4 \mathrm{H}, \mathrm{d}, J=7.0 \mathrm{~Hz}$, major $), 0.60(0.6 \mathrm{H}, \mathrm{d}, J=7.0$ $\mathrm{Hz}$, minor), $0.71(2.4 \mathrm{H}, \mathrm{d}, J=7.0 \mathrm{~Hz}$, major), $0.78(0.6 \mathrm{H}, \mathrm{d}, J=7.0 \mathrm{~Hz}$, minor), 0.78-0.91 (5H, m), 0.95-1.02 (1H, m), 1.28-1.33 (1H, m), 1.40-1.46 (1H, m), 1.58-1.67 (3H, m), 1.83-1.86 (1H, m), 2.72-2.89 (2H, m), $4.67(1 \mathrm{H}, \mathrm{ddd}, J=4.6,11.0,11.0 \mathrm{~Hz}), 4.85(1 \mathrm{H}, \mathrm{m}), 4.91(0.8 \mathrm{H}, \mathrm{m}$, major $), 5.14$ $(0.2 \mathrm{H}, \mathrm{m}, \mathrm{minor}), 6.54(2 \mathrm{H}, \mathrm{d}, J=8.6 \mathrm{~Hz}), 7.31-7.36(7 \mathrm{H}, \mathrm{m}) .{ }^{13} \mathrm{C}$ NMR: 15.7 (major), 16.0 (minor), 20.5 (major), 20.6 (minor), 21.9, 23.0 (major), 23.1 (minor), 25.8 (major), 25.9 (minor), 31.3 (major), 31.3 (minor), 34.0, 40.6(minor), 40.8 (major), 42.7 (minor), 43.4 (major), 46.7 (minor), 46.9 (major), 54.5 (minor), 54.7 (major), 74.9, 112.6, 119.2 (q, ${ }^{2} J_{\mathrm{C}-\mathrm{F}}=31.9 \mathrm{~Hz}$ ), 124.9 (q, ${ }^{1} J_{\mathrm{C}-\mathrm{F}}=268.6 \mathrm{~Hz}$ ), 126.1, $126.5\left(\mathrm{q},{ }^{3} J_{\mathrm{C}-\mathrm{F}}=3.1 \mathrm{~Hz}\right.$ ), 127.7 (minor), 127.8 (major), 128.9 (minor), 128.9 (major), 141.1 (minor), 141.2 (major), 149.2 (major), 149.3 (minor), 170.5 (major), 170.8 (minor). IR (nujol): 3350, 1705. EIMS $m / z$ : $447\left(\mathrm{M}^{+}\right)$. Anal. Calcd for $\mathrm{C}_{26} \mathrm{H}_{32} \mathrm{~F}_{3} \mathrm{NO}_{2}$ : C, 69.78; $\mathrm{H}$, $7.21 ; \mathrm{N}, 3.13$. Found: C, 69.68; H, 7.37; N, 3.21.

\section{$(1 R, 2 S, 5 R)$-2-Isopropyl-5-methylcyclohexyl 3-[4-(isopropoxycarbonyl)phenyl-} amino]-3-phenylpropanoate (4d):

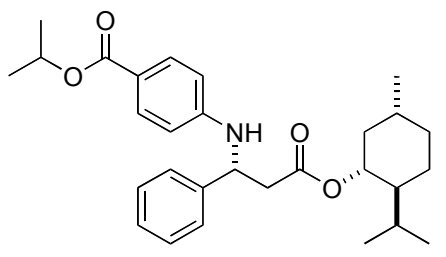

a diastereomeric mixture $(44 \%$ de $)$ as colorless needles of $\mathrm{mp}$ 153-155 ${ }^{\circ} \mathrm{C}$ and $[\alpha]^{25} \mathrm{D}-32.1$ (c 1.15, $\mathrm{CHCl}_{3}$ ). ${ }^{1} \mathrm{H}$ NMR: $0.57(2.16 \mathrm{H}, \mathrm{d}, J=7.0 \mathrm{~Hz}$, major), 0.59 $(0.84 \mathrm{H}, \mathrm{d}, J=7.0 \mathrm{~Hz}$, minor), $0.72(2.16 \mathrm{H}, \mathrm{d}, J=7.0 \mathrm{~Hz}$, major), $0.77(0.84 \mathrm{H}, \mathrm{d}, J=7.0 \mathrm{~Hz}$, minor), 0.77-0.92 (5H, m), 0.97-1.00 (1H, m), 1.26-1.30 (7H, m), 1.40-1.44 (1H, m), 1.49-1.54 (1H, m), 1.60-1.66 (2H, m), 1.82-1.85 (1H, m), $2.76(1 \mathrm{H}, \mathrm{dd}, J=8.2,14.4 \mathrm{~Hz}), 2,85(1 \mathrm{H}, \mathrm{dd}, J=5.5,14.4$ $\mathrm{Hz}), 4.67(1 \mathrm{H}, \mathrm{ddd}, J=4.3,10.7,10.7 \mathrm{~Hz}), 4.88(1 \mathrm{H}, \mathrm{m}), 5.01(0.72 \mathrm{H}, \mathrm{d}, J=7.4 \mathrm{~Hz}$, major $), 5.16$ $(1 \mathrm{H}, \mathrm{m}), 5.23(0.28 \mathrm{H}, \mathrm{d}, J=7.0 \mathrm{~Hz}$, minor $), 6.50(2 \mathrm{H}, \mathrm{d}, J=8.8 \mathrm{~Hz}), 7.23-7.25(1 \mathrm{H}, \mathrm{m}), 7.32(4 \mathrm{H}$, m), 7.79 (2H, d, $J=8.8 \mathrm{~Hz}) .{ }^{13} \mathrm{C}$ NMR: 15.9 (major), 16.0 (minor), 20.6 (major), 20.7 (minor), 21.9 (minor), 22.0 (major), 23.1 (major), 23.2 (minor), 25.9 (major), 26.0 (minor), 31.3, 34.1, 40.7 (minor), 40.8 (major), 42.9 (minor), 43.2 (major), 46.8 (minor), 46.9 (major), 54.4 (minor), 54.6 (major), 67.2, 74.8, 112.2, 119.5 (minor), 119.6 (major), 126.1, 127.5, (major), 127.6 (major), 128.8, 131.3, 141.0 (minor), 141.1 (major), 150.2 (major), 150.4 (minor), 166.2, 170.3 (major), 170.7 
(minor). IR (neat): 3350, 1700. EIMS m/z: $465\left(\mathrm{M}^{+}\right)$. Anal. Calcd for $\mathrm{C}_{29} \mathrm{H}_{39} \mathrm{NO}_{4}$ : C, 74.81; $\mathrm{H}, 8.44$; N, 3.01. Found: C, 74.55; H, 8.51; N, 2.88.

(1R,2S,5R)-2-Isopropyl-5-methylcyclohexyl 3-(4-cyanophenylamino)-3-phenylpropanoate (4e): $\mathrm{NC}$<smiles>CC1CCC(C(C)C)C(OC(=O)CC(Nc2ccccc2)c2ccccc2)C1</smiles>

a diastereomeric mixture $(20 \% \mathrm{de})$ as colorless needles of mp $119-127{ }^{\circ} \mathrm{C}$ and $[\alpha]^{25}{ }_{\mathrm{D}}-38.7\left(c\right.$ 0.99, $\left.\mathrm{CHCl}_{3}\right) .{ }^{1} \mathrm{H}$ NMR: $0.56(1.8 \mathrm{H}, \mathrm{d}, J=7.0 \mathrm{~Hz}$, major $), 0.58(1.2 \mathrm{H}, \mathrm{d}, J=7.0$ $\mathrm{Hz}$, minor), $0.72(1.8 \mathrm{H}, \mathrm{d}, J=7.0 \mathrm{~Hz}$, major), $0.77(1.2 \mathrm{H}, \mathrm{d}, J=7.0 \mathrm{~Hz}$, minor), 0.80-0.89 (5H, m), 0.95-1.02 (1H, m), 1.24-1.32 (1H, m), 1.43-1.47 (1H, m), 1.55-1.67 (3H, m), 1.80-1.86 (1H, m), $2.75(0.6 \mathrm{H}, \mathrm{dd}, J=8.6,14.7 \mathrm{~Hz}$, major), $2.79(0.4 \mathrm{H}, \mathrm{dd}, J=7.3,14.7 \mathrm{~Hz}$, minor $), 2.86(0.6 \mathrm{H}, \mathrm{dd}, J$ $=4.9,14.7 \mathrm{~Hz}$, major $), 2.88(0.4 \mathrm{H}, \mathrm{dd}, J=4.9,14.7 \mathrm{~Hz}$, minor $), 4.64(0.4 \mathrm{H}$, ddd, $J=4.3,11.1,11.1$ $\mathrm{Hz}$, minor), $4.66(0.6 \mathrm{H}$, ddd, $J=4.3,11.1,11.1 \mathrm{~Hz}$, major $), 4.84(1 \mathrm{H}, \mathrm{m}), 5.15(1 \mathrm{H}, \mathrm{d}, J=6.4 \mathrm{~Hz}$, major), $5.43\left(1 \mathrm{H}, \mathrm{d}, J=6.8 \mathrm{~Hz}\right.$, minor), $6.51(2 \mathrm{H}, \mathrm{d}, J=8.8 \mathrm{~Hz}), 7.33(7 \mathrm{H}, \mathrm{m}) .{ }^{13} \mathrm{C}$ NMR: 15.8 (major), 16.1 (minor), 20.6 (major), 20.7 (minor), 21.9, 23.1 (major), 23.2 (minor), 25.9, 31.3, 34.0, 40.7 (minor), 40.8 (major), 42.5 (minor), 43.2 (major), 46.7 (minor), 46.9 (major), 54.3 (minor), 54.6 (major), 75.0, 99.3, 113.0, 120.2, 126.0, 127.8, 128.9, 133.6, 140.4, 149.9 (major), 150.0 (minor), 170.6. IR (nujol): 3380, 2200, 1700. EIMS m/z: $404\left(\mathrm{M}^{+}\right)$. Anal. Calcd. for $\mathrm{C}_{26} \mathrm{H}_{32} \mathrm{~N}_{2} \mathrm{O}_{2}$ : C, 77.19; H, 7.97; N, 6.92. Found: C, 76.95; H, 8.00; N, 6.82.

\section{(1R,2S,5R)-2-Isopropyl-5-methylcyclohexyl 3-(4-nitrophenylamino)-3-phenylpropanoate (4f):}

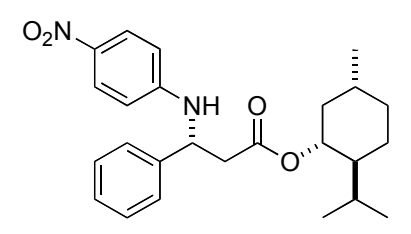

a diastereomeric mixture $(14 \%$ de) as pale yellow needles of $\mathrm{mp}$ 100-114 ${ }^{\circ} \mathrm{C}$ and $[\alpha]^{25}$ D $-49.3\left(c\right.$ 1.19, $\left.\mathrm{CHCl}_{3}\right) .{ }^{1} \mathrm{H}$ NMR: 0.57 (1.7H, d, $J=7.0 \mathrm{~Hz}$, major), 0.58 $(1.3 \mathrm{H}, \mathrm{d}, J=7.0 \mathrm{~Hz}$, minor), $0.73(1.7 \mathrm{H}, \mathrm{d}, J=7.0 \mathrm{~Hz}$, major), $0.76(1.3 \mathrm{H}, \mathrm{d}, J=7.0 \mathrm{~Hz}$, minor), 0.79-0.91 (5H, m), 0.95-1.04 (1H, m), 1.23-1.32 (1H, m), 1.38-1.47 (1H, m), 1.57-1.67 (3H, m), 1.80-1.86 (1H, m), 2.76-2.94 (2H, m), 4.62-4.70 (1H, m), 4.88-4.94 (1H, m), $5.49(1 \mathrm{H}, \mathrm{d}, J=6.4$ $\mathrm{Hz}), 5.79(1 \mathrm{H}, \mathrm{d}, J=6.8 \mathrm{~Hz}), 6.50(2 \mathrm{H}, \mathrm{d}, J=8.8 \mathrm{~Hz}), 7.27-7.35(5 \mathrm{H}, \mathrm{m}), 8.02(2 \mathrm{H}, \mathrm{d}, J=8.8 \mathrm{~Hz})$. ${ }^{13} \mathrm{C}$ NMR 15.9 (minor), 16.0 (major), 20.6 (minor), 20.7 (major), 23.1 (minor), 23.2 (major), 26.0 (major), 26.0 (minor), 31.3, 34.0, 40.7 (major), 40.8 (minor), 42.2 (major), 42.9 (minor), 46.7 (major), 46.9 (minor), 54.4 (major), 54.7 (minor), 75.1, 111.9, 126.0 (major), 126.1 (minor), 126.2, 127.9, 128.9, 138.3 (major), 138.4 (minor), 140.1 (major), 140.2 (minor), 151.9 (minor), 152.0 (major), 170.2 (minor), 170.6 (major). IR (nujol): 3350, 1710, 1500, 1330. EIMS m/z: $425\left(\mathrm{M}^{+}\right)$. Anal. Calcd for $\mathrm{C}_{25} \mathrm{H}_{32} \mathrm{~N}_{2} \mathrm{O}_{4}$ : C, 70.73; H, 7.60; N, 6.60. Found: C, 71.00; H, 7.82; N, 6.68. 
(4g):

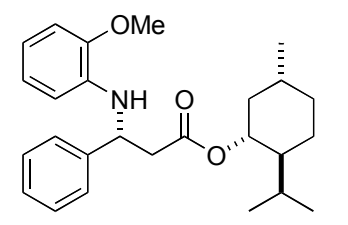

a diastereomeric mixture $\left(68 \%\right.$ de) as colorless amorphous of mp $83-94{ }^{\circ} \mathrm{C}$ and $[\alpha]^{25}{ }_{\mathrm{D}}-50.8\left(c \mathrm{1.13}, \mathrm{CHCl}_{3}\right) .{ }^{1} \mathrm{H}$ NMR: $0.54(2.5 \mathrm{H}, \mathrm{d}, J=6.7 \mathrm{~Hz}), 0.63(0.5 \mathrm{H}, \mathrm{d}, J=6.7 \mathrm{~Hz}), 0.70$ $(2.5 \mathrm{H}, \mathrm{d}, J=6.7 \mathrm{~Hz}), 0.79(0.5 \mathrm{H}, \mathrm{d}, J=6.7 \mathrm{~Hz}), 0.81(1 \mathrm{H}, \mathrm{m}), 0.88(3 \mathrm{H}, \mathrm{d}, J=7.0 \mathrm{~Hz}), .0 .93(1 \mathrm{H}$, m), $0.97(1 \mathrm{H}, \mathrm{m}), 1.26(1 \mathrm{H}, \mathrm{m}), 1.43(1 \mathrm{H}, \mathrm{m}), 1.59-1.66(3 \mathrm{H}, \mathrm{m}), 1.88-1.91(1 \mathrm{H}, \mathrm{m}), 2.81(2 \mathrm{H}, \mathrm{d}, J$ $=7.4 \mathrm{~Hz}), 3.86(2.5 \mathrm{H}, \mathrm{s}), 3.87(0.5 \mathrm{H}, \mathrm{s}), 4.66(1 \mathrm{H}, \mathrm{ddd}, J=4.3,11,11 \mathrm{~Hz}), 4.83(1 \mathrm{H}, \mathrm{dt}, J=6.1$, $7.4 \mathrm{~Hz}), 5.00(1 \mathrm{H}, \mathrm{d}, J=6.1 \mathrm{~Hz}), 6.38(1 \mathrm{H}, \mathrm{m}), 6.61(1 \mathrm{H}, \mathrm{m}), 6.69-6.75(2 \mathrm{H}, \mathrm{m}), 7.21-7.32(3 \mathrm{H}$, m), 7.38-7.39 (2H, m). ${ }^{13} \mathrm{C}$ NMR: 15.8, 20.7, 22.0, 23.1, 25.8, 31.3, 34.2, 40.8, 43.8, 47.0, 54.9, 55.4, 74.5, 109.2, 110.8, 116.6, 121.0, 126.3, 126.3, 127.3, 128.6, 128.6, 136.5, 142.3, 146.7, 170.5 . IR (nujol): 1710, 3400. EIMS m/z: $409\left(\mathrm{M}^{+}\right)$. Anal. Calcd for $\mathrm{C}_{26} \mathrm{H}_{35} \mathrm{NO}_{3}$ : C, 76.25; H, 8.61; N, 3.42. Found: C, 76.51; H, 8.46; N, 3.43.

\section{$(1 R, 2 S, 5 R)-2-$ Isopropyl-5-methylcyclohexyl}

\section{3-(4-methoxyphenylamino)-3-[4-(trifluoromethyl)phenyl]propanoate (4h):}<smiles>COc1ccc(NCC(=O)OC2CC(C)CCC2C(C)C)cc1</smiles>

recrystallization of a diastereomeric mixture $(94 \%$ de) from $\mathrm{MeOH}$ gave a pure major isomer as colorless needles of mp $141.0-142.0{ }^{\circ} \mathrm{C}$ and $[\alpha]^{25}{ }_{\mathrm{D}}-25.0\left(c 1.00, \mathrm{CHCl}_{3}\right) .{ }^{1} \mathrm{H}$ NMR: $0.57(3 \mathrm{H}, \mathrm{d}, J=7.0 \mathrm{~Hz}), 0.72(3 \mathrm{H}, \mathrm{d}, J=7.0 \mathrm{~Hz}), 0.78-0.90(5 \mathrm{H}, \mathrm{m}), 0.94-1.03(1 \mathrm{H}, \mathrm{m})$, 1.23-1.34 (1H, m), 1.37-1.50 (1H, m), 1.52-1.69 $(3 \mathrm{H}, \mathrm{m}), 1.77-1.88(1 \mathrm{H}, \mathrm{m}), 2.72-2.82(2 \mathrm{H}, \mathrm{m})$, $3.67(3 \mathrm{H}, \mathrm{s}), 4.36(1 \mathrm{H}, \mathrm{br}), 4.65(1 \mathrm{H}, \mathrm{ddd}, J=4.3,10.7,10.7 \mathrm{~Hz}), 4.81(1 \mathrm{H}, \mathrm{m}), 6.47(2 \mathrm{H}, \mathrm{d}, J=8.9$ $\mathrm{Hz}), 6.69(2 \mathrm{H}, \mathrm{d}, J=8.9 \mathrm{~Hz}), 7.50(2 \mathrm{H}, \mathrm{d}, J=7.9 \mathrm{~Hz}), 7.57(2 \mathrm{H}, \mathrm{d}, J=7.9 \mathrm{~Hz}) .{ }^{13} \mathrm{C} \mathrm{NMR}: 15.8$, $20.5,21.8,23.1,25.9,31.3,34.0,40.8,43.2,46.9,55.5,55.6,74.8,114.8,114.9,124.1\left(\mathrm{q},{ }^{1} J_{\mathrm{C}-\mathrm{F}}=\right.$ $270.7 \mathrm{~Hz}), 125.7,\left(\mathrm{q},{ }^{3} J_{\mathrm{C}-\mathrm{F}}=4.1 \mathrm{~Hz}\right), 126.8,129.7\left(\mathrm{q},{ }^{2} J_{\mathrm{C}-\mathrm{F}}=31.9 \mathrm{~Hz}\right), 140.4,146.6,152.5,170.4$. IR (neat): 3402, 1709. EIMS m/z: $477\left(\mathrm{M}^{+}\right), 339,280$. Anal. Calcd for $\mathrm{C}_{27} \mathrm{H}_{34} \mathrm{~F}_{3} \mathrm{NO}_{3}$ : C, 67.91; $\mathrm{H}$, 7.18; N, 2.93. Found: C, 67.87; H, 7.07; N, 2.96.

\section{(1R,2S,5R)-2-Isopropyl-5-methylcyclohexyl}

\section{3-(4-methoxyphenylamino)-3-(4-methoxyphenyl)propanoate (4i):}

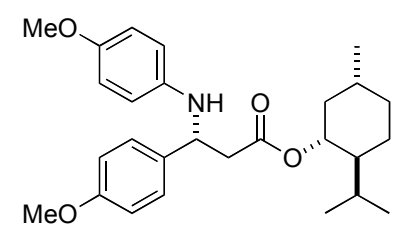

recrystallization of a diastereomeric mixture $(92 \% \mathrm{de})$ from $\mathrm{EtOH}$ gave a pure major isomer as colorless needles of mp $120.5-121{ }^{\circ} \mathrm{C}$ and $[\alpha]^{25} \mathrm{D}-28.7\left(c 1.00, \mathrm{CHCl}_{3}\right) .{ }^{1} \mathrm{H}$ NMR: $0.57(3 \mathrm{H}, \mathrm{d}, J=7.0 \mathrm{~Hz}), 0.73(3 \mathrm{H}, \mathrm{d}, J=7.0 \mathrm{~Hz}), 0.78-1.02(6 \mathrm{H}, \mathrm{m}), 1.26-1.32(1 \mathrm{H}, \mathrm{m})$, 
1.40-1.46 (1H, m), 1.58-1.66 (3H, m), 1.86-1.88 (1H, m), 2.73-2.75 (2H, m), 3.69 (3H, s), 3.78 $(3 \mathrm{H}, \mathrm{s}), 4.18(1 \mathrm{H}, \mathrm{br}), 4.62-4.67(1 \mathrm{H}, \mathrm{ddd}, J=4.3,10.7,10.7 \mathrm{~Hz}), 4.69-4.71(1 \mathrm{H}, \mathrm{m}), 6.50(2 \mathrm{H}, \mathrm{d}$, $J=8.9 \mathrm{~Hz}), 6.69(2 \mathrm{H}, \mathrm{d}, J=8.9 \mathrm{~Hz}), 6.84(2 \mathrm{H}, \mathrm{d}, J=8.6 \mathrm{~Hz}), 7.28(2 \mathrm{H}, \mathrm{d}, J=8.6 \mathrm{~Hz}) .{ }^{13} \mathrm{C} \mathrm{NMR:}$ 15.8, 20.6, 21.9, 23.1, 25.8, 31.1, 34.1, 40.9, 43.6, 46.9, 55.2, 55.3, 55.7, 74.5, 114.1, 114.7, 114.9, 127.5, 134.4, 141.0, 152.2, 158.9, 170.9. IR (neat): 3387, 1717. EIMS $m / z: 439\left(\mathrm{M}^{+}\right), 242,123$. Anal. Calcd for $\mathrm{C}_{27} \mathrm{H}_{37} \mathrm{NO}_{4}$ : C, 73.77; H, 8.48; N, 3.19. Found: C, 73.56; H, 8.51; N, 2.99.

\section{$(1 R, 2 S, 5 R)$-2-Isopropyl-5-methylcyclohexyl}

\section{3-(4-methoxyphenylamino)-3-(naphthalen-1-yl)propanoate $(4 \mathbf{j})$ :}

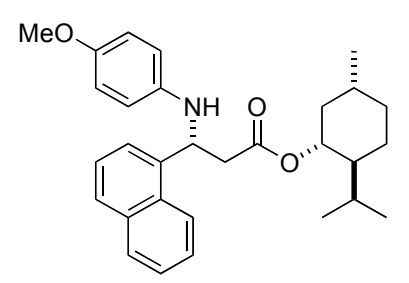

recrystallization of a diastereomeric mixture $(64 \%$ de) from $\mathrm{MeOH}$ gave a diastereomeric mixture $(1 \% \mathrm{de})$ as colorless needles of mp $122-123{ }^{\circ} \mathrm{C}$ and $[\alpha]^{25}{ }_{\mathrm{D}}-57.4(c 1.00$, $\left.\mathrm{CHCl}_{3}\right) .{ }^{1} \mathrm{H}$ NMR: $0.58(3 \mathrm{H}, \mathrm{d}, J=7.0 \mathrm{~Hz}), 0.72(3 \mathrm{H}, \mathrm{d}, J=7.0 \mathrm{~Hz}), 0.81-0.91(5 \mathrm{H}, \mathrm{m}), 0.98-1.03$ (1H, m), 1.26-1.36 (1H, m), 1.42-1.46 (1H, m), 1.61-1.64 (2H, m), 1.69-1.73 (1H, m), 1.86-1.92 $(1 \mathrm{H}, \mathrm{m}), 2.72(0.5 \mathrm{H}, \mathrm{dd}, J=8.5,14.7 \mathrm{~Hz}$, major), $2.82(0.5 \mathrm{H}, \mathrm{dd}, J=8.3,14.7 \mathrm{~Hz}, \operatorname{minor}), 3.04(1 \mathrm{H}$, m), $3.67(3 \mathrm{H}, \mathrm{s}), 4.42(1 \mathrm{H}, \mathrm{br}), 4.70(1 \mathrm{H}, \mathrm{ddd}, J=4.3,11,11 \mathrm{~Hz}), 5.55(1 \mathrm{H}, \mathrm{m}), 6.44(1 \mathrm{H}, \mathrm{d}, J=8.8$ $\mathrm{Hz}$, major), $6.48(1 \mathrm{H}, \mathrm{d}, J=8.8 \mathrm{~Hz}$, minor), $6.64(1 \mathrm{H}, \mathrm{d}, \mathrm{J}=8.8 \mathrm{~Hz}$, major), $6.67(1 \mathrm{H}, \mathrm{d}, J=8.8 \mathrm{~Hz}$, minor), $7.39(1 \mathrm{H}, \mathrm{t}, J=7.6 \mathrm{~Hz}), 7.50-7.54(1 \mathrm{H}, \mathrm{m}), 7.57-7.63(2 \mathrm{H}, \mathrm{m}), 7.76(1 \mathrm{H}, \mathrm{d}, J=8.3 \mathrm{~Hz})$, $7.91(1 \mathrm{H}, \mathrm{d}, J=8.3 \mathrm{~Hz}), 8.21(1 \mathrm{H}, \mathrm{d}, J=8.6 \mathrm{~Hz}) .{ }^{13} \mathrm{C}$ NMR: 15.8 (major), 16.0 (minor), 20.6, 21.9 23.1 (minor), 23.2 (major), 26.0, 31.3, 34.1, 40.7 (minor), 40.9 (major), 41.9 (minor), 42.5 (major), 46.8 (minor), 47.0 (major), 51.9, 52.1, 55.7, 74.8, 114.5 (minor), 114.6 (major), 114.8, 122.3 (major), 122.4 (minor), 123.2, 125.6, 125.8, 126.4, 128.0, 129.2 (minor), 129.3 (major), 130.6 (major), 130.7 (minor), 134.2, 137.1 (major), 137.2 (minor), 140.9 (major), 141.0 (minor), 152.2, 171.1 (minor), 171.2 (major). IR (neat): 1713, 3341. EIMS $m / z: 459\left(\mathrm{M}^{+}\right)$. Anal. Calcd for $\mathrm{C}_{30} \mathrm{H}_{37} \mathrm{NO}_{3}$ : C, 78.40; H, 8.11; N, 3.05. Found: C, 78.25; H, 8.01; N, 3.03.

\section{$(1 R, 2 S, 5 R)$-2-Isopropyl-5-methylcyclohexyl}

\section{3-(4-methoxyphenylamino)-3-(naphthalen-2-yl)propanoate (4k):}

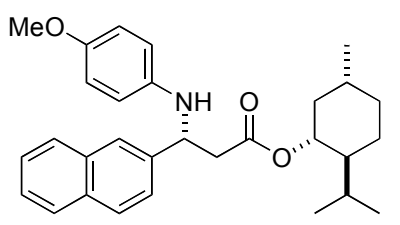

recrystallization of a diastereomeric mixture $(88 \%$ de) from $\mathrm{MeOH}$ gave a pure major isomer as colorless needles of $\mathrm{mp} 116.0-117.0{ }^{\circ} \mathrm{C}$ and $[\alpha]^{25}{ }_{\mathrm{D}}-18.1\left(c 1.00, \mathrm{CHCl}_{3}\right) .{ }^{1} \mathrm{H}$ NMR: $0.50(3 \mathrm{H}, \mathrm{d}, J=7.0 \mathrm{~Hz}), 0.64(3 \mathrm{H}, \mathrm{d}, J=7.0 \mathrm{~Hz}), 0.76-0.86(5 \mathrm{H}, \mathrm{m}), 0.92-1.00(1 \mathrm{H}, \mathrm{m})$, $1.24-1.29(1 \mathrm{H}, \mathrm{m}), 1.41-1.46(1 \mathrm{H}, \mathrm{m}), 1.50-1.54(1 \mathrm{H}, \mathrm{m}), 1.59-1.64(2 \mathrm{H}, \mathrm{m}), 1.82-1.84(1 \mathrm{H}, \mathrm{m})$, 2.81-2.89 (2H, m), $3.67(3 \mathrm{H}, \mathrm{s}), 4.34(1 \mathrm{H}, \mathrm{br}), 4.64(1 \mathrm{H}, \mathrm{ddd}, J=4.3,11,11 \mathrm{~Hz}), 4.91(1 \mathrm{H}, \mathrm{m})$, 
$6.54(2 \mathrm{H}, \mathrm{d}, J=8.8 \mathrm{~Hz}), 6.67(2 \mathrm{H}, \mathrm{d}, J=8.8 \mathrm{~Hz}), 7.42-7.45(2 \mathrm{H}, \mathrm{m}), 7.50(1 \mathrm{H}, \mathrm{d}, J=8.6 \mathrm{~Hz})$, 7.77-7.83 (4H, m). ${ }^{13} \mathrm{C}$ NMR: 15.7, 20.5, 21.9, 23.0, 25.8, 31.3, 34.1, 40.8, 43.4, 46.9, 55.7, 56.2, 74.6, 114.8, 114.9, 124.4, 125.3, 125.8, 126.1, 127.7, 128.0, 128.6, 133.0, 133.5, 139.8, 140.9, 152.3, 170.8. IR (neat): 1717, 3387. EIMS $m / z: 459\left(\mathrm{M}^{+}\right)$. Anal. Calcd for $\mathrm{C}_{30} \mathrm{H}_{37} \mathrm{NO}_{3}: \mathrm{C}, 78.40 ; \mathrm{H}$, 8.11; N, 3.05. Found: C, 78.21; H, 8.35; N, 2.96.

\section{Methyl (R)-(+)-3-(4-methoxyphenylamino)-3-phenylpropanoate (Scheme 3):}

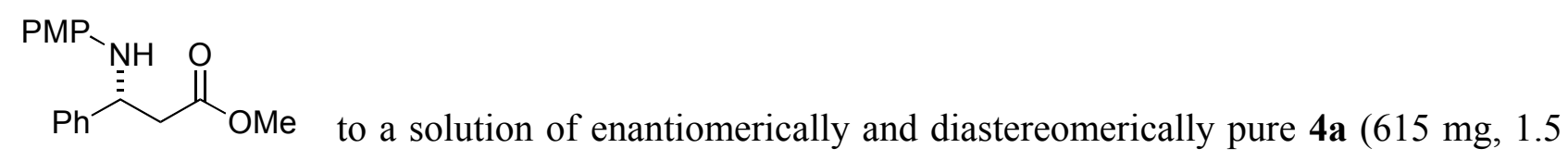
mmol; recrystallized 4a with $96: 4 \mathrm{dr}$ in $70 \%$ recovery from methanol) in dry THF (3.0 mL) was added dropwise a THF $(3.0 \mathrm{~mL})$ solution of anhydrous $n-\mathrm{Bu}_{4} \mathrm{NOH}$, prepared from a 40 wt \% aqueous solution $(1.95 \mathrm{~g}, 3.0 \mathrm{mmol})$ by azeotropic removal of water with DME $(7.5 \mathrm{~mL})$ and toluene $(7.5 \mathrm{~mL})$ twice. After stirred at $0{ }^{\circ} \mathrm{C}$ for $1 \mathrm{~h}$, the mixture was quenched with $3 \mathrm{~N} \mathrm{HCl}(10$ $\mathrm{mL})$ and extracted with EtOAc $(100+50+25 \mathrm{~mL})$. The combined organic layers were dried and concentrated. The resulting crude mixture was dissolved in $\mathrm{MeOH}(5 \mathrm{~mL})$, and a $1 \mathrm{M}$ hexane solution of $\mathrm{Me}_{3} \mathrm{SiCHN}_{2}$ was added to the solution at room temperature. The mixture was stirred until gas evolution ceased, and subsequently quenched with $\mathrm{HCO}_{2} \mathrm{H}$ until no more $\mathrm{N}_{2}$ evolution was observed. The mixture was diluted with EtOAc $(125 \mathrm{~mL})$, washed with water $(50 \mathrm{~mL})$, sat. $\mathrm{NaHCO}_{3}(50 \mathrm{~mL} \times 2)$, and brine $(50 \mathrm{~mL})$, and then dried. Concentration gave a brown oil (630 mg). Chromatography (hexane/EtOAc $=9 / 1)$ gave the titled methyl ester $(320 \mathrm{mg}, 75 \%)$ as colorless needles of $\mathrm{mp} 69.0-70.5{ }^{\circ} \mathrm{C}$ and $[\alpha]^{25}+8.1\left(c 1.03, \mathrm{CHCl}_{3}\right) .{ }^{1} \mathrm{H}$ NMR: $2.78(1 \mathrm{H}, \mathrm{dd}, J=6.7,15.3$ $\mathrm{Hz}), 2.82(1 \mathrm{H}, \mathrm{dd}, J=6.7,15.3 \mathrm{~Hz}), 3.65(3 \mathrm{H}, \mathrm{s}), 3.69(3 \mathrm{H}, \mathrm{s}), 4.27(1 \mathrm{H}, \mathrm{bs}), 4.75(1 \mathrm{H}, \mathrm{dd}, J=6.7$, 6.7 Hz), 6.51-6.54 (2H, m), 6.65-6.71 (2H, m), 7.24 (1H, m), 7.30-7.37 (4H, m). ${ }^{13} \mathrm{C}$ NMR: 42.7, 51.8, 55.6, 55.8, 114.7, 115.2, 126.2, 127.4, 128.7, 140.9, 142.4, 152.3, 171.6. IR (nujol): 1712, 3368. EIMS $m / z$ : $285\left(\mathrm{M}^{+}\right)$. Anal. Calcd for $\mathrm{C}_{17} \mathrm{H}_{19} \mathrm{NO}_{3}$ : C, 71.56; H, 6.71; N, 4.91. Found: C, 71.44; H, 6.60; N, 4.87.

\section{Methyl (R)-(+)-3-amino-3-phenylpropanoate (7):}

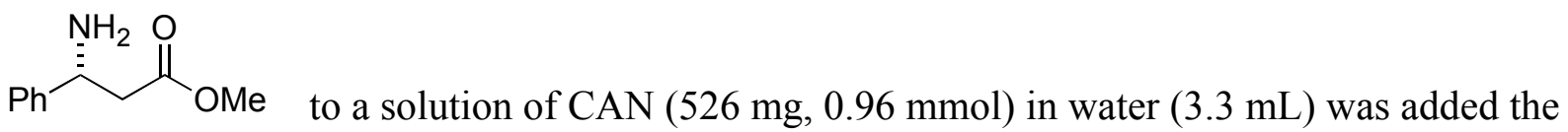
above methyl ester $(68 \mathrm{mg}, 0.24 \mathrm{mmol})$ in $\mathrm{MeCN}(2.5 \mathrm{~mL})$ at $0{ }^{\circ} \mathrm{C}$ over $10 \mathrm{~min}$. The mixture was stirred for $1 \mathrm{~h}$, and water $(5 \mathrm{~mL})$ was added. The mixture was washed with $\mathrm{Et}_{2} \mathrm{O}(25+15 \mathrm{~mL})$, and $\mathrm{Na}_{2} \mathrm{CO}_{3}$ was added to the aqueous layer until its $\mathrm{pH}$ became 7 . The mixture was filtrated through a pad of celite, and the filtrate was extracted with EtOAc $(20 \mathrm{~mL}$ x 3). The combined organic layers were dried, concentrated, and purified by chromatography (EtOAc) to give 7 (21 mg, 49\%) as a brown oil of $[\alpha]^{26}{ }_{\mathrm{D}}+20.9\left(c 1.05, \mathrm{CHCl}_{3}\right):{ }^{1} \mathrm{H}$ NMR: $1.90(2 \mathrm{H}, \mathrm{bs}), 2.67(2 \mathrm{H}, \mathrm{d}, J=7.0 \mathrm{~Hz}), 3.68$ $(3 \mathrm{H}, \mathrm{s}), 4.42(1 \mathrm{H}, \mathrm{t}, J=7.0 \mathrm{~Hz}), 7.25-7.27(1 \mathrm{H}, \mathrm{m}), 7.32-7.36(4 \mathrm{H}, \mathrm{m}) .{ }^{13} \mathrm{C} \mathrm{NMR}: 43.9,51.6,52.6$, 126.1, 127.4, 128.6, 144.5, 172.4. IR (nujol): 1740, 3350. EIMS $m / z: 179\left(\mathrm{M}^{+}\right)$. 
$4 a$

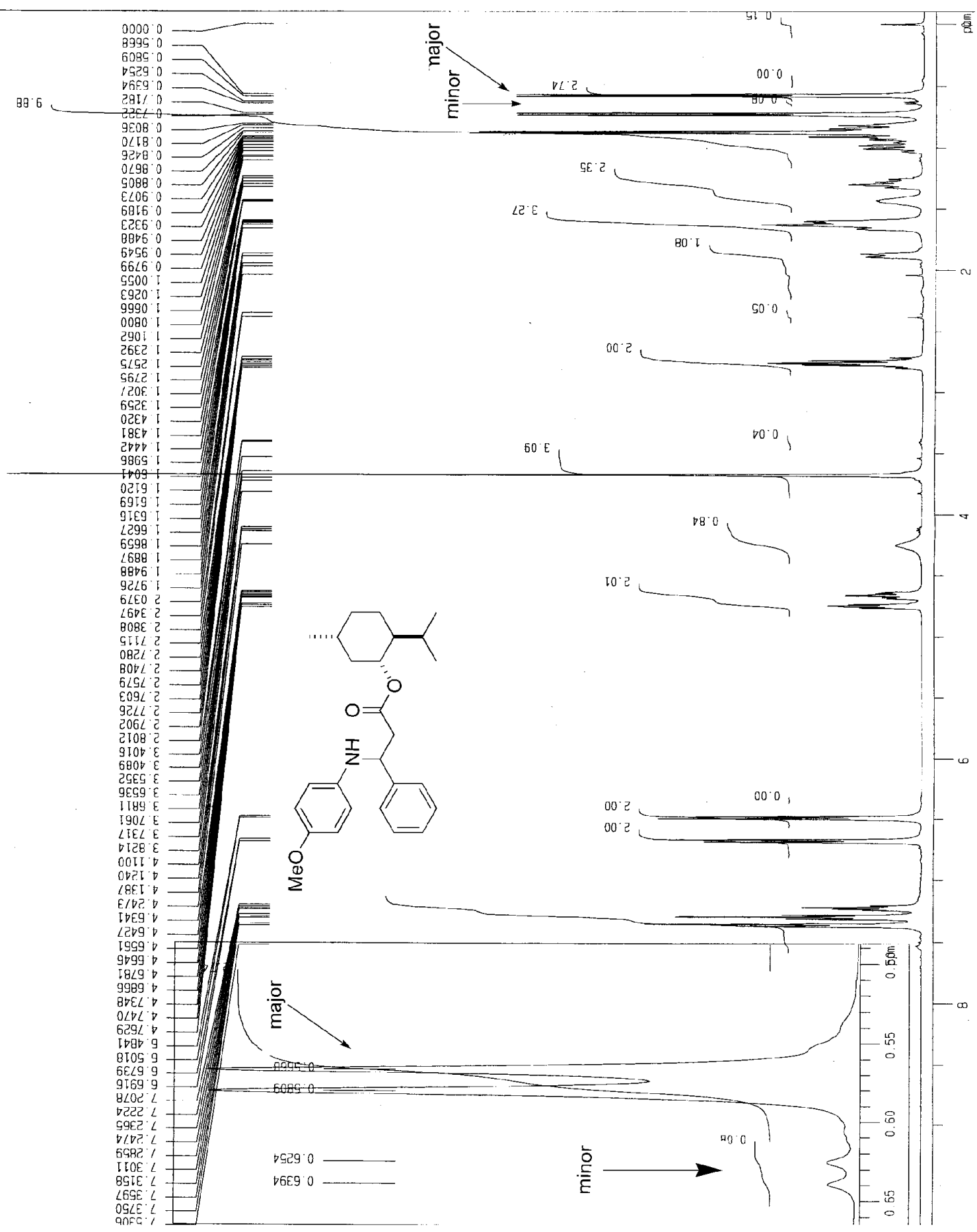




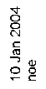

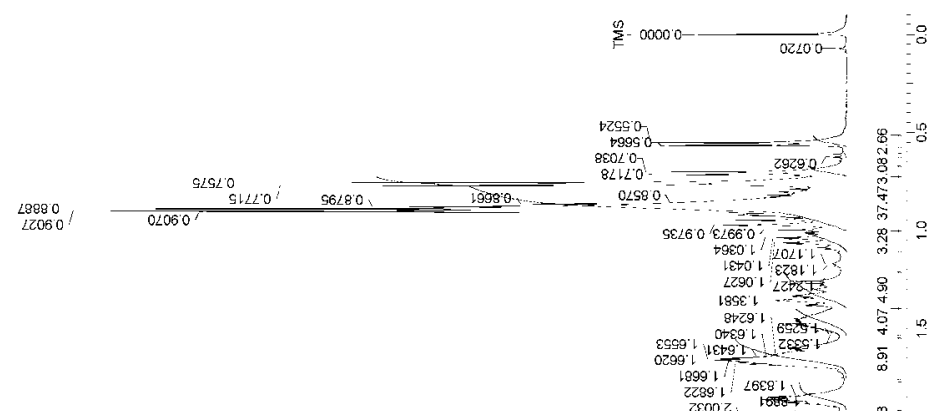

$\subseteq \check{Z} 0 己$

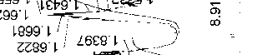

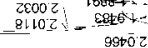

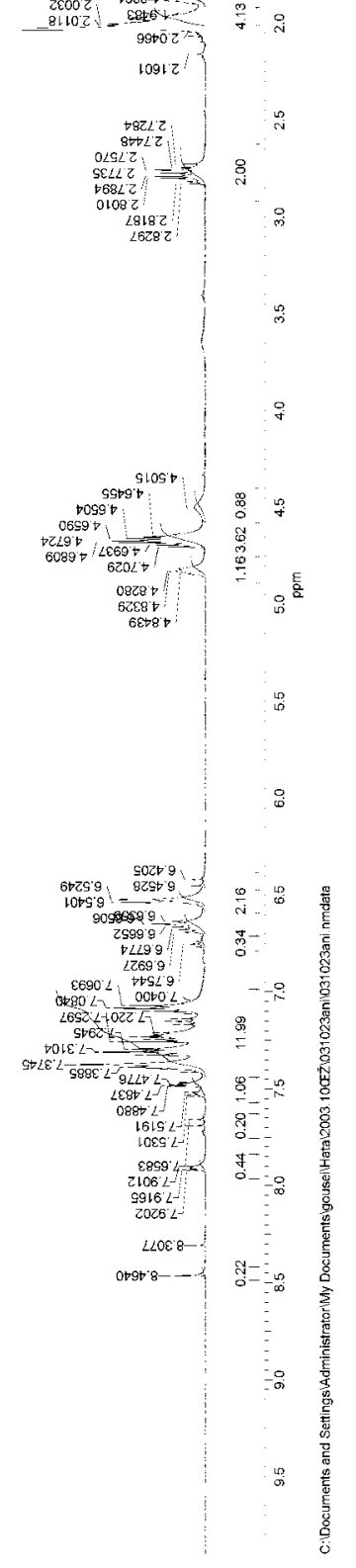

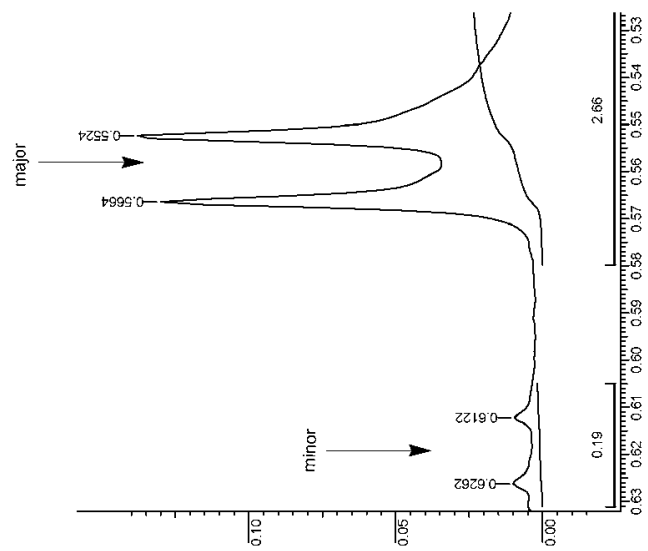

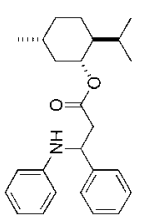




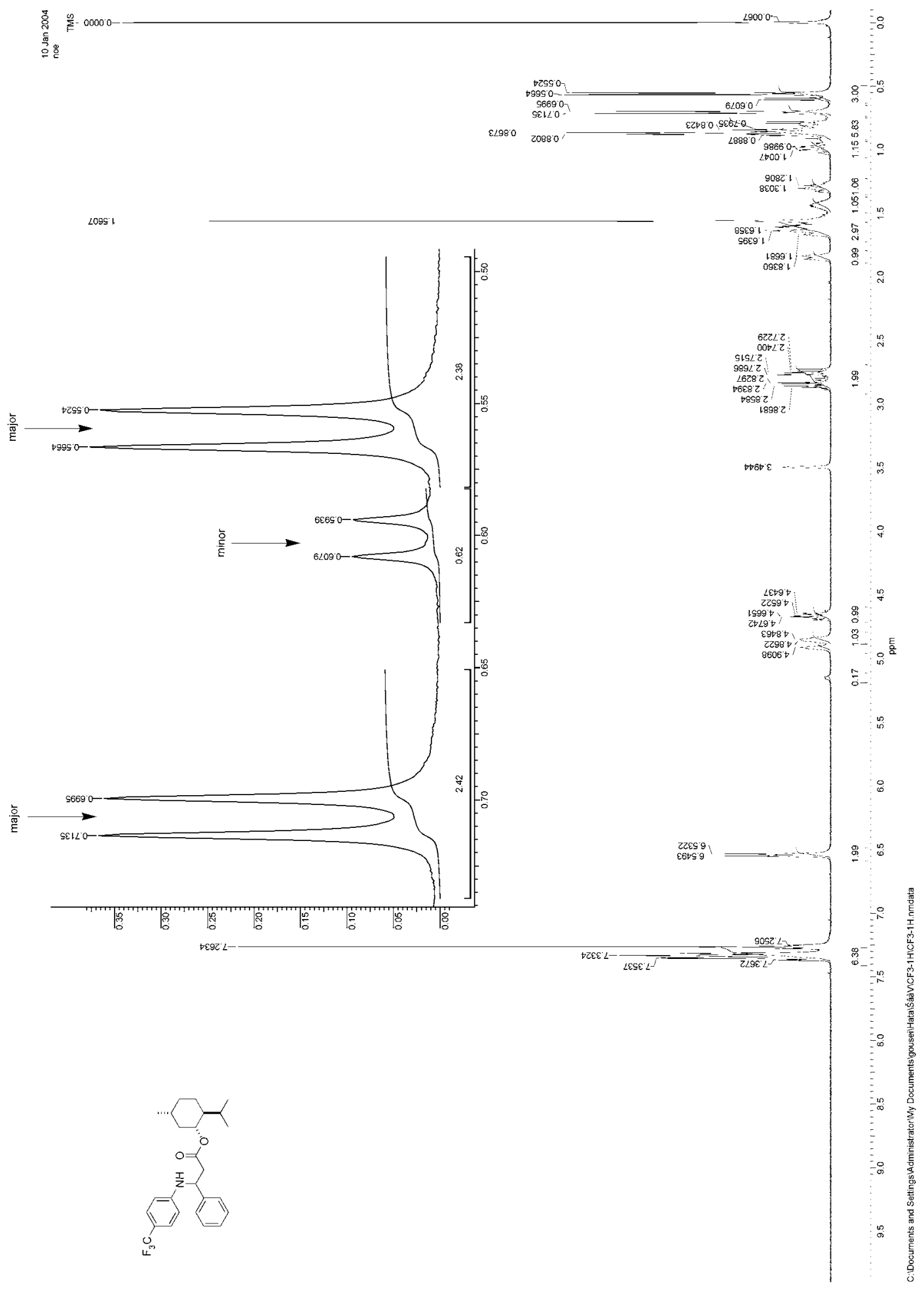




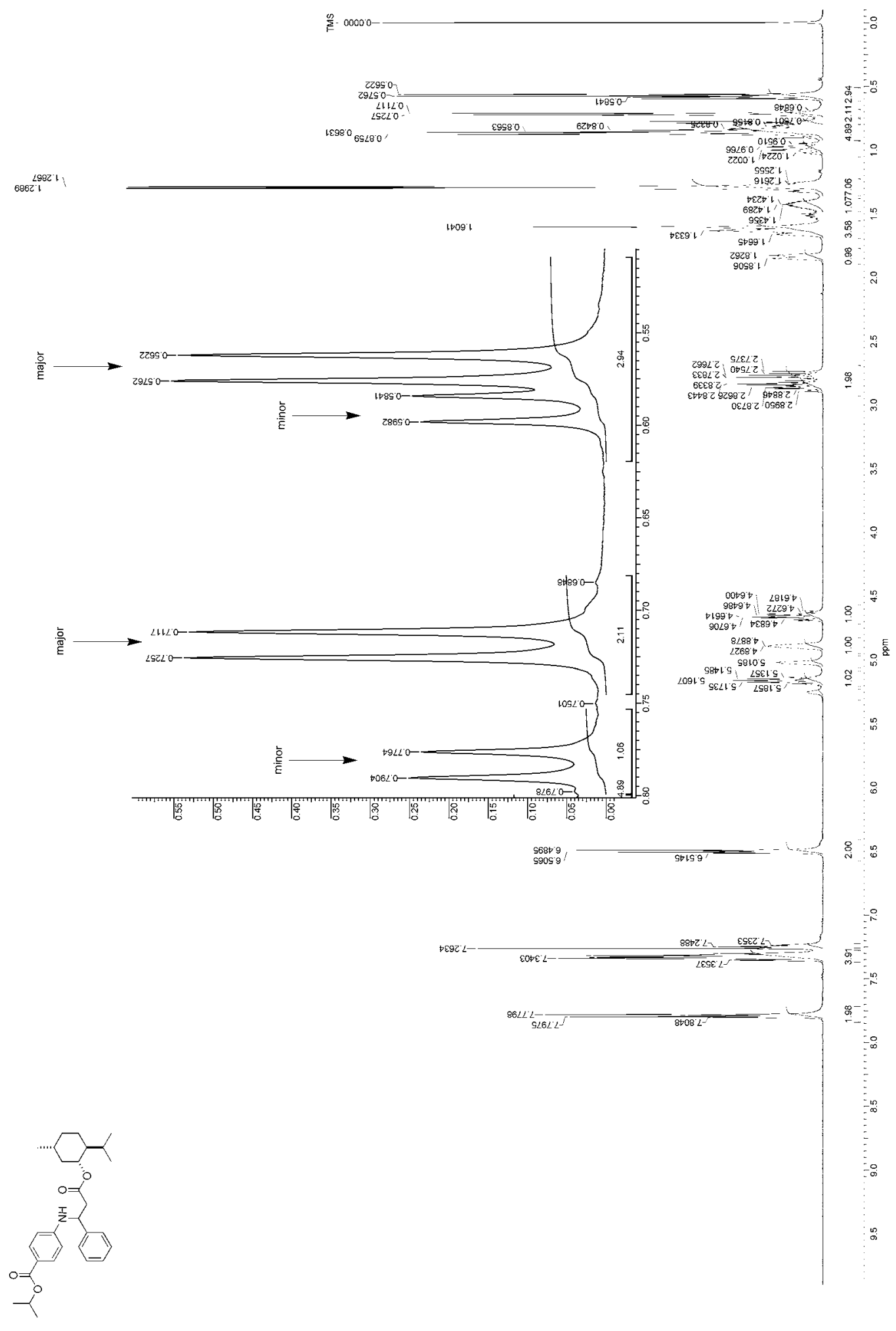




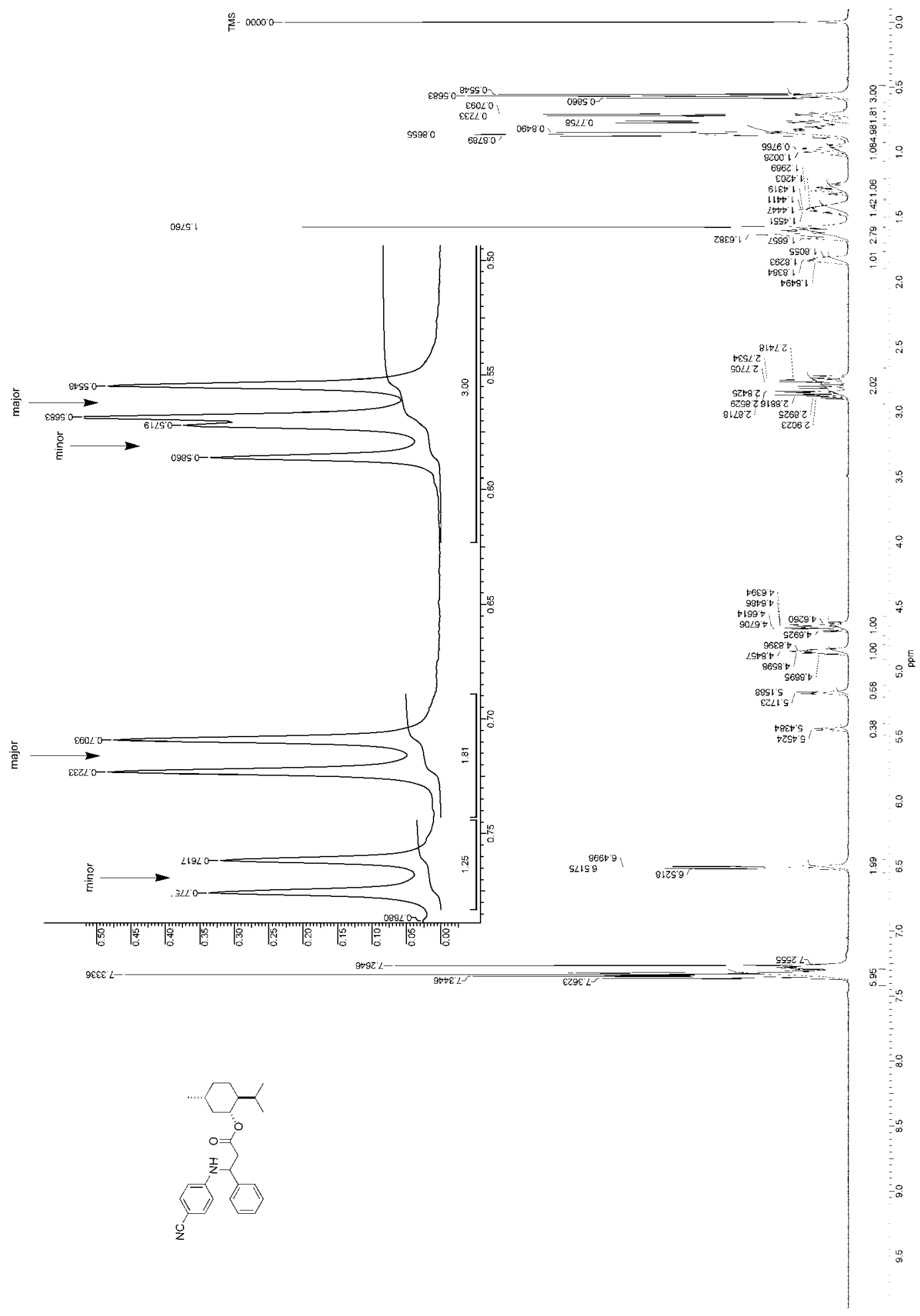




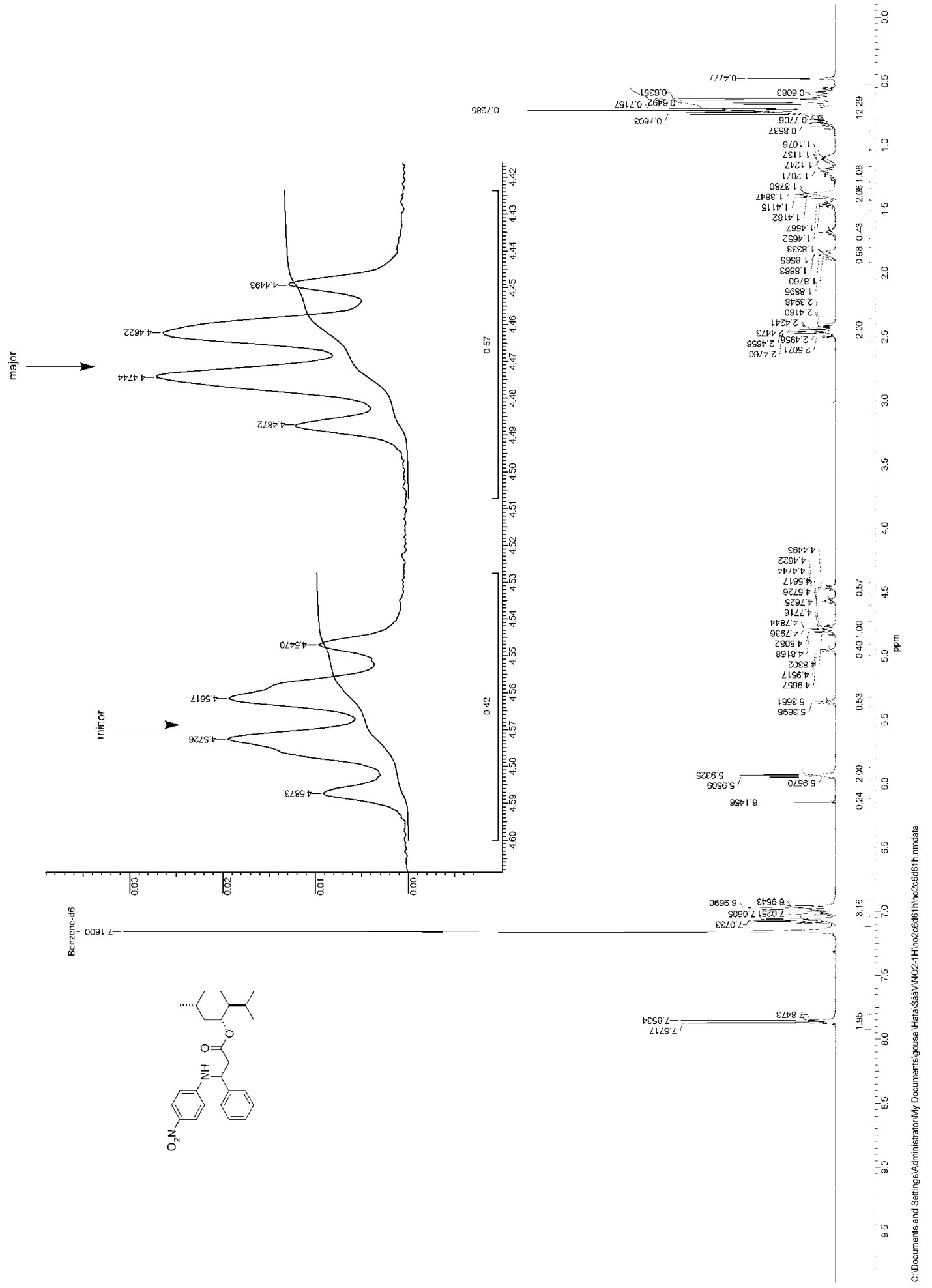




\section{$4 g$}

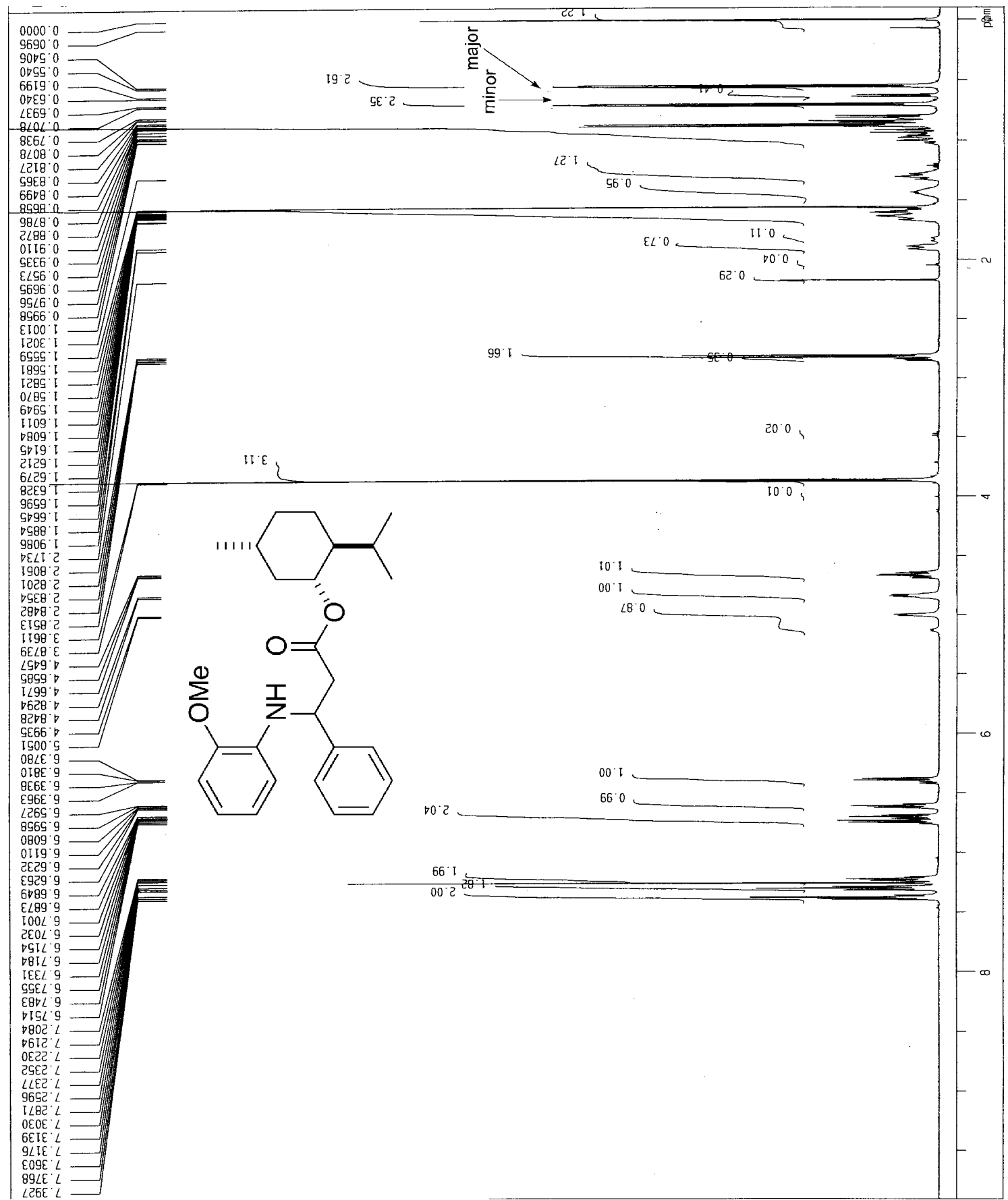




\section{4h}

De was determinied by crude ${ }^{13} \mathrm{C}-\mathrm{NMR}$.

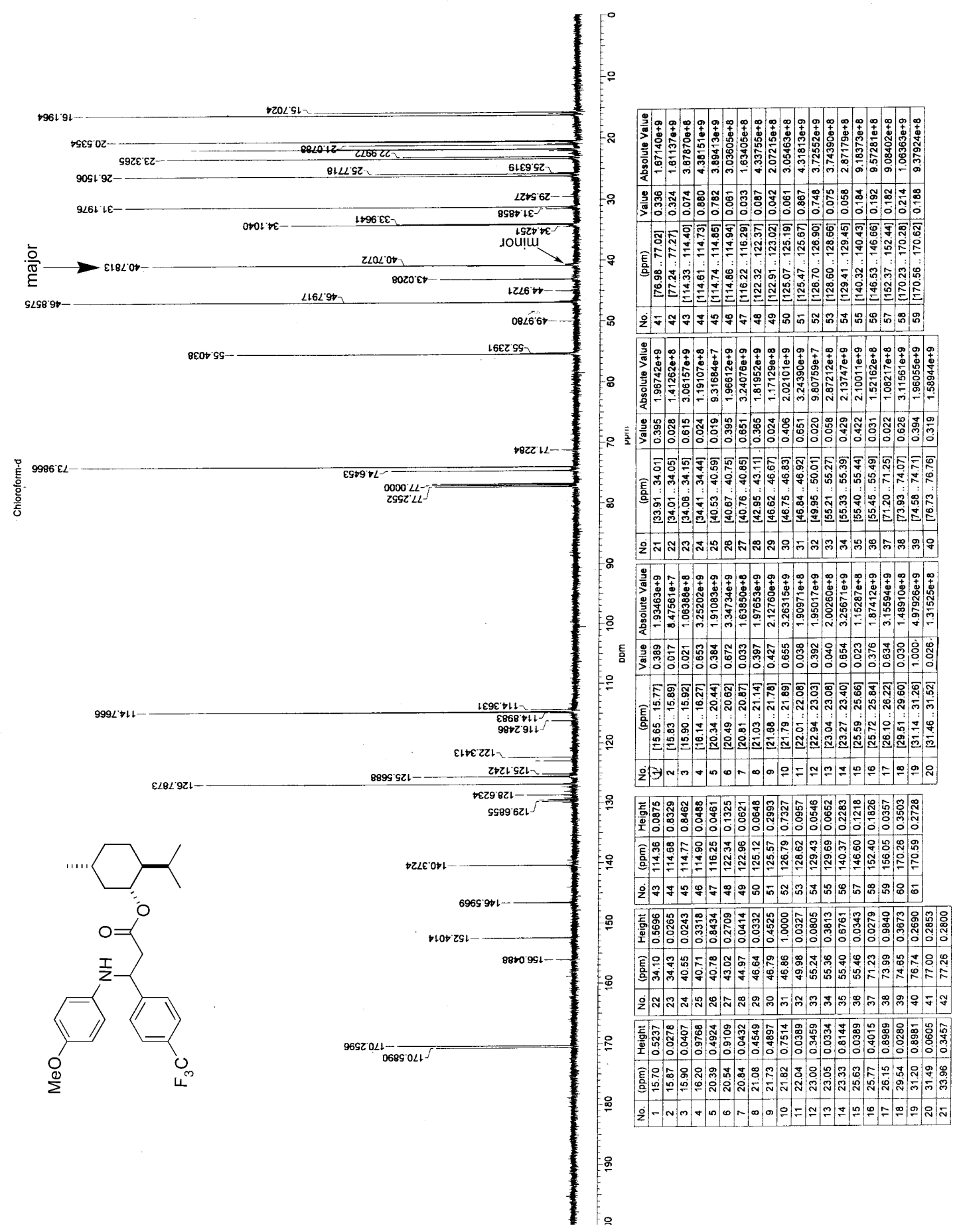


De was determinied by crude ${ }^{1} \mathrm{H}-\mathrm{NMR}$.

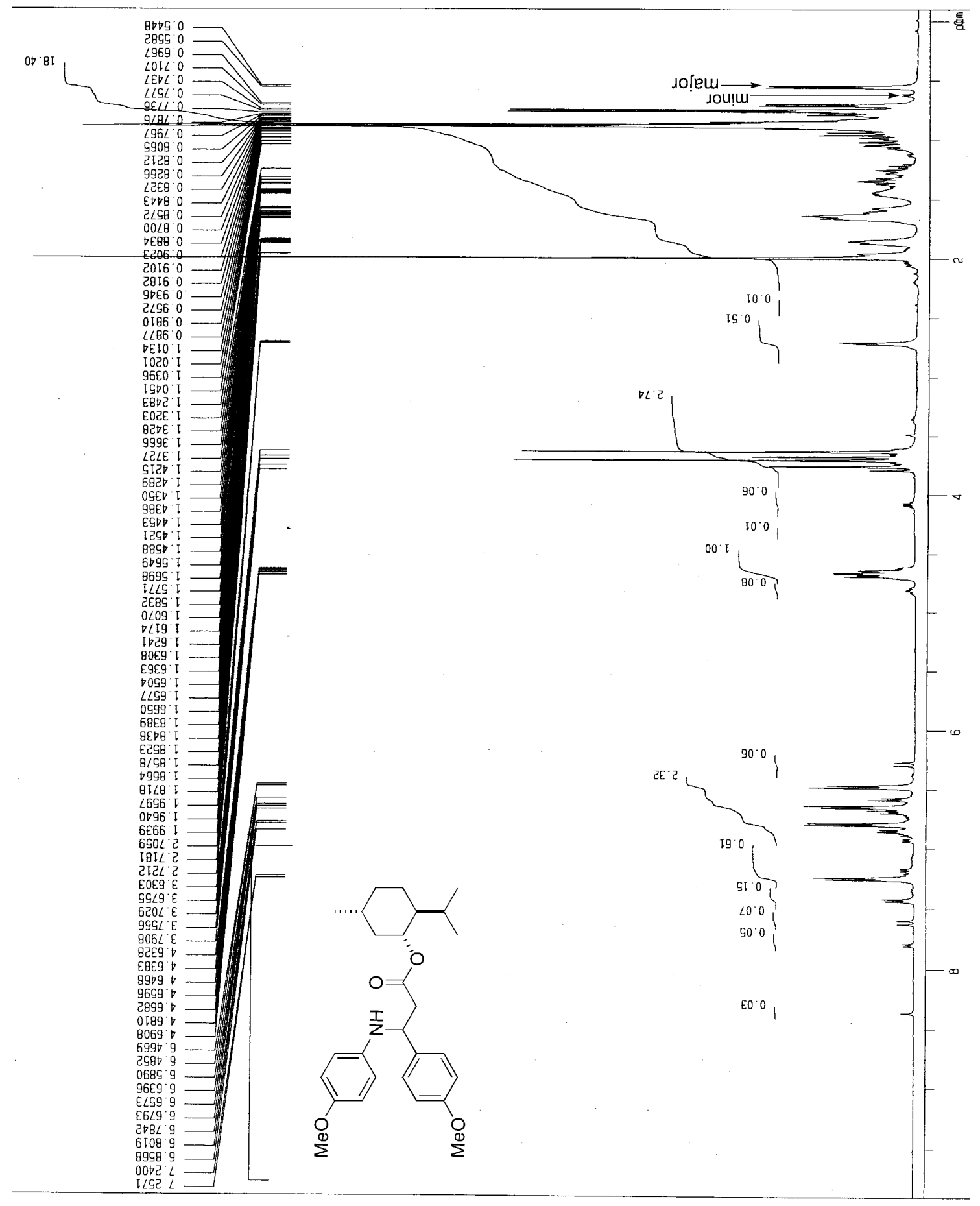




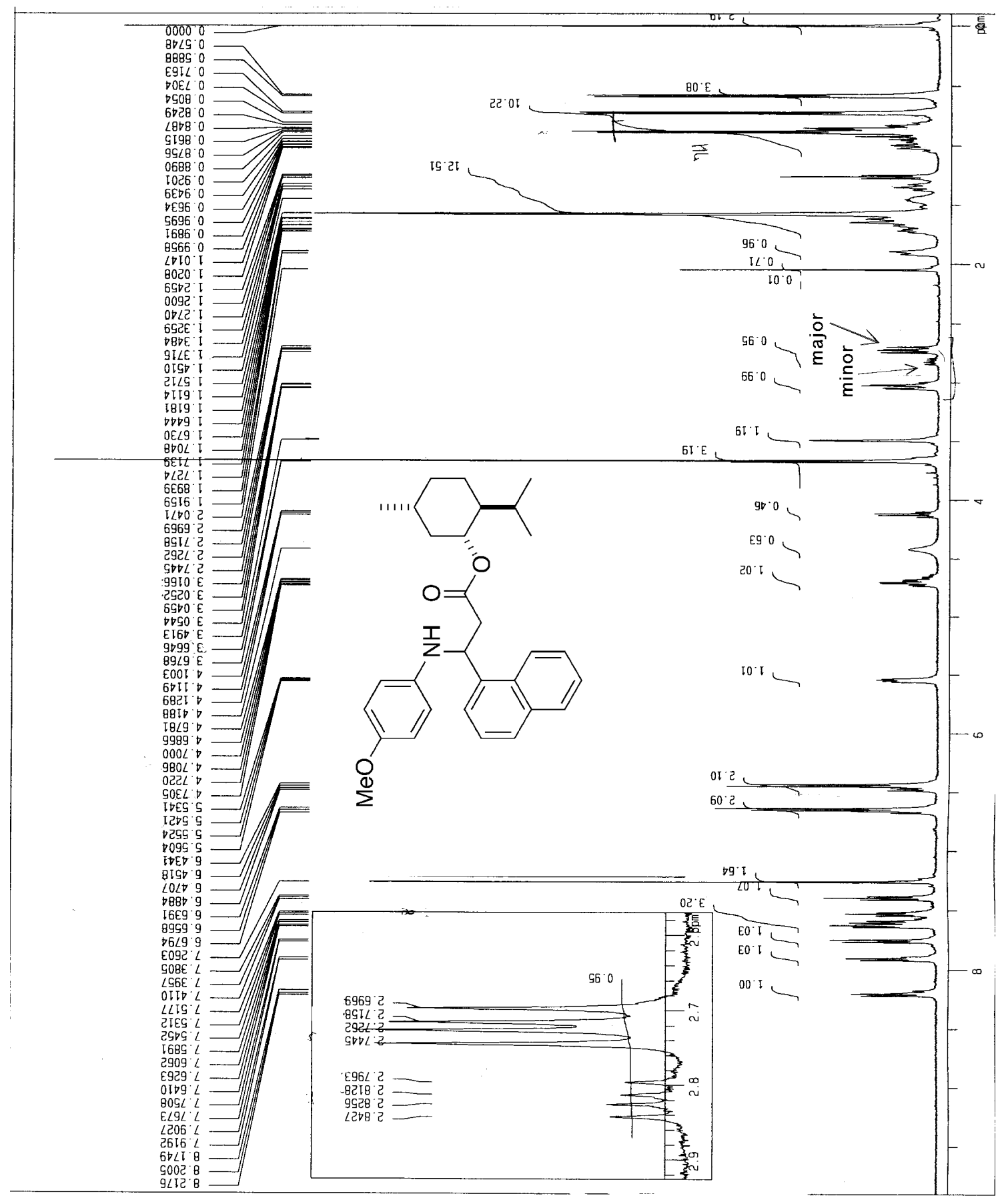




\section{4k (DMSO-d6)}

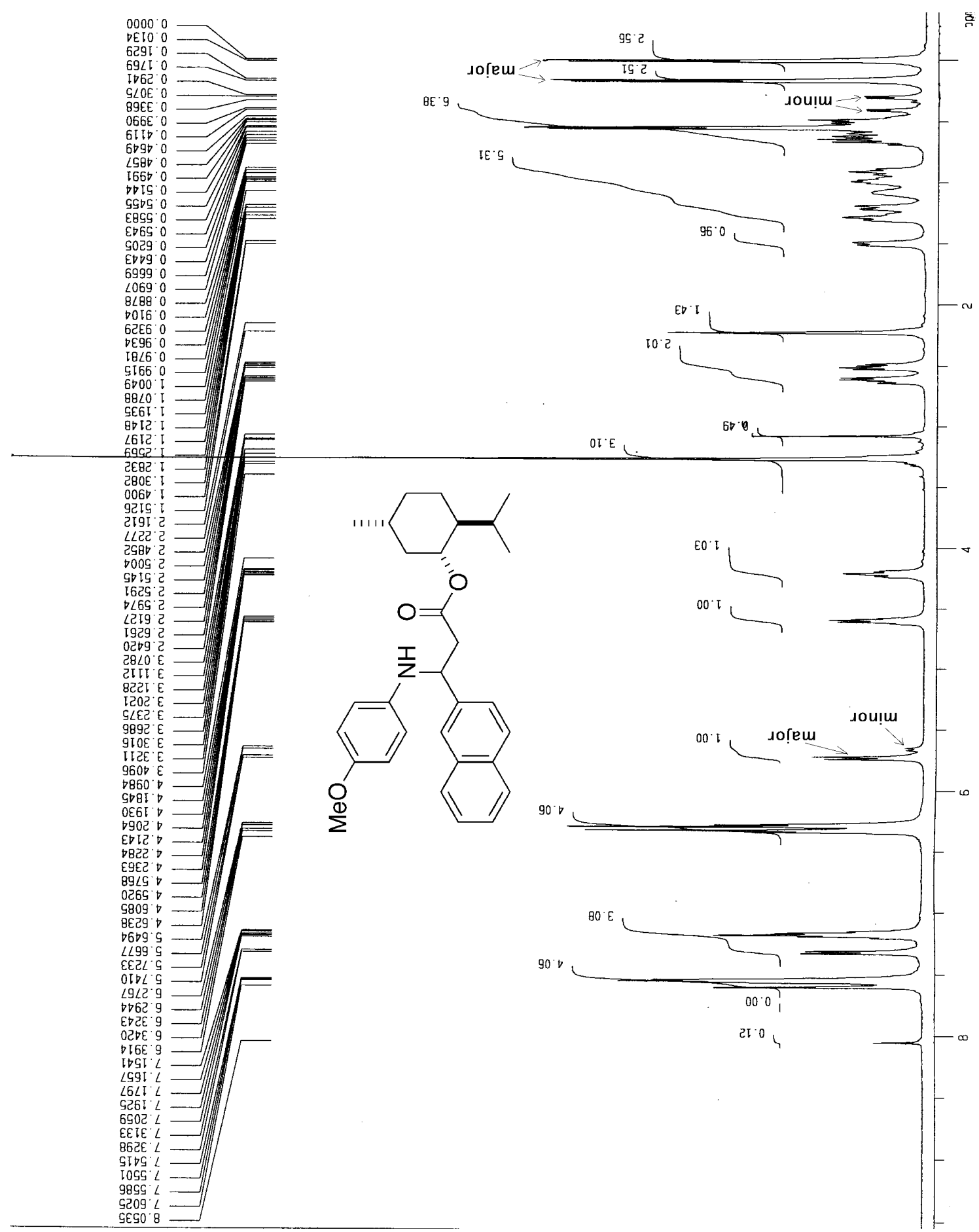

